\title{
Treatment by Transarterial Chemoembolization and Sorafenib for Hepatocellular Carcinoma vs Advanced Hepatocellular Carcinoma in Bangladesh: A Real-life Situation
}

\author{
Mamun Al Mahtab ${ }^{1}$, Md Abdur Rahim², Sheikh Mohammad Noor-E-Alam³, Mohammad Ashraful Alam ${ }^{4}$, \\ Faiz Ahmad Khondaker ${ }^{5}$, Ahmed Lutful Moben ${ }^{6}$, Jhumur Ghosh7, Md Sakirul Islam Khan ${ }^{8}$
}

\begin{abstract}
Background: Although there are thousands of patients with hepatocellular carcinoma (HCC) in Bangladesh, almost there is no nationwide recommendations for therapeutic maneuver for HCC patients. As most of the HCC patients of Bangladesh are diagnosed late, patients with advanced HCC with large and multiple HCC nodules represent the usual presentation of HCC. The study is presented here to assess the effects of a combined therapy of transarterial chemoembolization (TACE) and sorafenib in advanced HCC patients.

Materials and methods: A total of 77 patients with advanced HCC were enrolled in this study. All of them had advanced HCC with variable etiologies and clinical conditions. All patients were treated by TACE and also given systemic sorafenib at the same time. The patients were checked 1 and 3 months after therapy commencement.

Results: Out of 77 patients, 18 patients died within 1 month and additional 15 patients died within 3 months after therapy commencement. Thus, there remains 44 surviving patients after 3 months. When different parameters of dead patients and survived patients were compared, no specific parameters dictated the factors about overall survival of more than 3 months.

Discussion: This is the first approach to initiate an invasive and a systemic therapy for treatment of advanced HCC in Bangladesh. Further follow-up of patients and their long-term overall survival may cast some lights about the role of these therapies in Bangladeshi HCC patients. Keywords: Hepatocellular carcinoma, Sorafenib, Transarterial chemoembolization.

Euroasian Journal of Hepato-Gastroenterology (2019): 10.5005/jp-journals-10018-1300
\end{abstract}

\section{INTRODUCTION}

Hepatocellular carcinoma is the fourth most common cause of cancer-related death and about 1 million patients die each year due to $\mathrm{HCC}^{1,2}$ indicating the importance and implication of this pathological condition. The prevalence and incidence of HCC is global in nature, but there is marked variation regarding these parameters.

Although HCC has been a major public health problem for several decades, the prognosis of HCC was devastating before three decades with most patients dying within the first year after diagnosis irrespective of management strategies., ${ }^{3,4}$ Even, developed countries were not exception to this fundamental. However, some concurrent developments altered the scenario of HCC diagnosis and management tremendously during last-two to three decades. The first, with the emergence of standardized surveillance strategies and the introduction of the Barcelonaclinic liver cancer classification (BCLC) for clinical management of $\mathrm{HCC}$, significant improvement has been achieved regarding early diagnosis of HCC. The next, various new and novel treatment modalities have made revolutions in the management of HCC. Finally, drugs for systemic usage have added extrabenefit for management of advanced HCC. ${ }^{5}$ However, these developments are available in rich, advanced, and developed countries. As a matter of fact, the benefit of science for surveillance and management of HCC has not reached to general population of developing and resource-constrained countries. ${ }^{6}$

Bangladesh is a country of about 170 million people with hepatitis $B$ virus (HBV) carrier rate of $2-5 \%$ and hepatitis $C$ virus $(\mathrm{HCV})$ carrier
${ }^{1,3}$ Department of Hepatology, Bangabandhu Sheikh Mujib Medical University, Dhaka, Bangladesh

${ }^{2}$ Department of Hepatology, Abdul Malek Ukil Medical College, Noakhali, Bangladesh

${ }^{4}$ Department of Hepatology, Shaheed Tajuddin Ahmad Medical College, Gazipur, Bangladesh

${ }^{5}$ Department of Hepatology, Shaheed Suhrawardy Medical College, Dhaka, Bangladesh

${ }^{6}$ Department of Hepatology, Kurmitola General Hospital, Dhaka, Bangladesh

${ }^{7}$ Department of Medicine, MH Samorita Hospital and Medical College, Dhaka, Bangladesh

${ }^{8}$ Department of Anatomy and Embryology, Graduate School of Medicine, Ehime University, Toon, Ehime, Japan

Address reprint requests to: Mamun Al Mahtab, Department of Hepatology, Bangabandhu Sheikh Mujib Medical University, Dhaka, Bangladesh, Phone: +880 171156 7275, e-mail: shwapnil@agni.com

How to cite this article: Al Mahtab, M, Rahim MA, Noor-E-Alam SM, et al. Treatment by Transarterial Chemoembolization and Sorafenib for Hepatocellular Carcinoma vs Advanced Hepatocellular Carcinoma in Bangladesh: A Real-life Situation. Euroasian J Hepato-Gastroenterol 2019;9(2):63-66.

Source of support: Nil

Conflict of interest: None

rate of about $1 \% .^{7}$ Although nation-wise epidemiological data are not available about prevalence of HCC in Bangladesh, there seems 
to be several thousand patients with HCC in this country. However, a workable and standardized surveillance and management system of HCC is yet to be developed for Bangladesh. Although Bangladesh harbor about 5-8 million chronic HBV-infected persons and about 2 million HCV-infected subjects, less than $10 \%$ patients are aware of their HBV or HCV infectivity. A small percentage of population receive treatment for chronic hepatitis and there is no proper surveillance system for these subjects. In this pretext and socioeconomic status of Bangladesh, management of HCC remains a matter of personal choice and this is usually fixed on the basis of technical know-how and economical status of the physicians and patients. There is no national health insurance system at Bangladesh as well. For long, cirrhosis of liver (CL) and HCC, two major complications of chronic HBV and HCV infection, have been regarded as diseases of symptomatic management with almost no avenue for treatment in Bangladesh. Thus, the patients with HCC are diagnosed at the late stage of their illness and almost remain out of any available therapeutic approaches. A study has shown that the majority of HCC patients attend physicians at a size of more than $5 \mathrm{~cm}$ in diameter. ${ }^{3}$ Patients with solitary and small HCC rarely attend the physicians in Bangladesh. It is also a matter of great regret that there has been almost no publication and reports about management of HCC in Bangladesh.

The last two decades can be regarded as a notable time frame related to development of different therapies for HCC. There has been development of localized treatment for ablation of HCC nodules and also general treatment for containment of HCC. As a general term, TACE is most widely used for treatment of primary HCC in advanced countries. ${ }^{8-11}$ Transarterial chemoembolization has been used in some developed and advanced countries as a means to proceed the HCC patients to future liver transplantation. On the other hand, sorafenib is the only proven drug for systemic treatment for advanced HCC. Taken together, there remains a strong rationale to combine both treatment modalities for management of $\mathrm{HCC}$, especially advanced HCC, even if there remains no opportunity of liver transplantation-a reality of developing and resourceconstrained countries like Bangladesh.

Even with these developments for therapy of HCC patients, almost nothing is accomplished in HCC patients of Bangladesh except traditional medical management. Even if that is done, very limited numbers of patients can reach to those therapy.

The study presented here represents the first study to design systematic analysis of the clinical presentations of HCC at Bangladesh. Also, the effect of combined treatment with sorafenib and TACE in advanced HCC patients at Bangladesh was evaluated as a part of treatment of HCC in real-life situation. The long-term prognosis of these patients would provide more insights about HCC management at Bangladesh and possibly in other developing and resource-constrained countries.

\section{Materials and Methods}

\section{Patients}

The study has been designed as a single-tail study with no controlled arm, and thus no comparison with other modes of therapy was planned. In fact, a study with multiple wings is not possible in advanced HCC patients in a developing country like Bangladesh. The study was designed on the basis to achieve the study purpose. The study was conducted at one modernized hospital of Dhaka city, Bangladesh.

The therapy has been initiated in 150 patients with HCC, and 77 of these patients have completed an initial target of 3-months follow-up. For further analysis, the data of 77 patients would be shown. The age of the patients was $51.3 \pm 12.5$ years (range 24-75 years). Out of them, 62 were males and 15 females. The inclusion criteria were not formulated on the basis of numbers of SOL or the size of space occupying lesion (SOL). The patients were explained about the pros and cons of the therapy and the patients who gave consent were included in the study.

Parameters of biochemical assessment showed high degree of variability. For example, the levels of alpha feto protein (AFP) varied from $16 \mathrm{ng} / \mathrm{dL}$ to $>200,000 \mathrm{ng} / \mathrm{dL}$. The levels of alanine aminotransferase (ALT) varied from $19 \mathrm{IU} / \mathrm{L}$ to $134 \mathrm{IU} / \mathrm{L}$. The levels of serum albumin were $3.2 \pm 0.58 \mathrm{~g} / \mathrm{dL}$. Sixteen patients had ascites. Solitary HCC nodules were detected in 30 patients. The size of the nodules was mostly more than $5 \mathrm{~cm}$ in diameter except only in one case when it was $1.5 \mathrm{~cm}$ in diameter. Eleven patients had HCC nodules of $10 \mathrm{~cm}$ or more in diameter. The largest HCC nodule was $20.7 \mathrm{~cm}$ in diameter. In the rest 47 patients, there were multiple HCC nodules or diffuse nodules.

Regarding the etiology of HCC, the majority of the patients have been suffering from HBV-related HCC (51 out of 77 patients, 66.2\%). The HCCs of other 26 patients were due to HCV, non alcoholic steatohepatitis (NASH), cryptogenic HCC, or secondary liver cancer due to primary lesions in other organs. The clinical parameters at the time of commencement of the therapy of all 77 patients have been shown in Table 1.

\section{Methodology of TACE and Dose of Sorafenib}

Transarterial chemoembolization was performed according to the standard procedure.$^{8-10}$ Access to the right femoral artery was made in the right groin using the femoral sheath $[A 6 \mathrm{~F}(2.0 \mathrm{~mm}) 11 \mathrm{~cm}$ (MEDTRONIC, Minneapolis, MN, USA)]. Flushing was done by $10-20 \mathrm{~mL}$ of normal saline through a flushing channel. The following steps were adapted to accomplish the TACE. After approaching to the hepatic artery, epirubicin $50 \mathrm{mg}(10 \mathrm{~mL})$ plus $10 \mathrm{~mL}$ lipidol was introduced. Embolization was done by absolute gel (scratched spongy foam mixed with $5 \mathrm{~mL}$ normal saline). If SOL was very large or the feeding vessel cannot be reached, only chemotherapy was injected and embolization was not done. Some patients developed fever, nausea, and pain for 2-3 days. But those were managed by traditional therapy.

Table 1: Baseline data of all patients undergoing transarterial chemoembolization therapy

\begin{tabular}{ll}
\hline & Total $(n=77)$ \\
\hline Age (years) & $51.3 \pm 12.5$ \\
Male : female & $62: 15$ \\
Etiology & \\
HBV & 51 \\
HCV & 11 \\
NASH & 5 \\
Cryptogenic & 4 \\
Others & 6 \\
Bilirubin (mg/dL) & $1.34 \pm 1.56$ \\
Protein (g/dL) & $3.20 \pm 0.57$ \\
AFP (ng/dL) & $27,980 \pm 151,661$ \\
ALT (IU/L) & $60.6 \pm 32.8$ \\
INR & $1.25 \pm 0.21$ \\
PT & $15.56 \pm 2.60$ \\
\hline
\end{tabular}

The data of 77 patients with advanced HCC whose follow-up of therapy for more than 3 months was accomplished have been shown 


\section{Results}

The study was designed to develop insights about the HCC status of Bangladeshi patients at their initial presentation. The next target was to assess the impacts of the combined therapy with TACE and sorafenib on advanced HCC at Bangladesh. The primary end point was the survival for 3 months after start of therapy. A total of 77 patients with HCC were subjected to the combined therapy and follow-up for 3 months was possible.

Out of total 77 patients with HCC, 33 patients died within 3 months of the observation period. Out of these 33 deaths, 18 patients died within 1 month and 15 patients died within 3 months. A total of 44 patients with HCC have been surviving for more than 3 months.

To develop insight regarding factors that may be related to death, we analyzed the baseline data of dead and surviving patients. Some notable data were retrieved from this study. All patients with diffuse HCC died within 3 months. Also, the patients with HBV-related HCC died more frequently than patients with other etiologies. Out of 77 patients, 51 were HBV-related HCC. Out of these 51 patients, 24 died (41\%) within 3 months; on the other hand, nine patients with other etiologies died within this period. The levels of bilirubin were slightly higher at entry in dead patients compared to survived patients, but the difference was not significant (Table 2).

As shown in Table 3, the baseline data of patients who survived for more than 3 months were analyzed. No specific factor emerged from this analysis.

\section{Discussion}

A lack of nationwide epidemiological data does not point to any specific figure of HCC prevalence in Bangladesh. Even, a conservative epidemiological assessment states that there may be several thousands of HCC patients in this country. The number may not be growing at any specific point as similar numbers of patients have been dying due to HCC.

There is no surveillance system for HCC or LC or CHB in Bangladesh and possibly in most developing and resourceconstrained countries. Thus, diagnosis is delayed and treatment is limited to conservative management of terminal HCC. This strongly contrasts to rich, advanced, and developed countries of the world, where, a strong surveillance system for HCC and its

Table 2: Baseline data of the dead and survived patients with hepatocellular carcinoma

\begin{tabular}{lll}
\hline & Survived $(n=44)$ & Dead $(n=33)$ \\
\hline Age (years) & $51 \pm 34$ & $51.7 \pm 12.5$ \\
Sex (male : female) & $38: 6$ & $24: 9$ \\
Etiology & & \\
$\quad$ HBV & 27 & 24 \\
HCV & 9 & 2 \\
NASH & 3 & 1 \\
$\quad$ Cryptogenic & 3 & 2 \\
$\quad$ Others & & 4 \\
Bilirubin (mg/dL) & $1.16 \pm 0.92$ & $1.68 \pm 2.34$ \\
Protein (g/dL) & $3.26 \pm 0.54$ & $3.1 \pm 0.61$ \\
AFP (ng/dL) & $38,433 \pm 178,729$ & $1,444 \pm 2,570$ \\
ALT (IU/L) & $56.5 \pm 33.2$ & $68.2 \pm 3,105$ \\
INR & $1.25 \pm 0.21$ & $1.24 \pm 0.221$ \\
PT & $15.46 \pm 2.8$ & $15.71 \pm 2.2$ \\
\hline
\end{tabular}

Table 3: Data of survived patients for more than 3 months due to therapy

\begin{tabular}{lll}
\hline & Baseline $(n=77)$ & $\begin{array}{l}3 \text { months } \\
\text { survived }(n=44)\end{array}$ \\
\hline Age & $51.3 \pm 12.5$ & $51 \pm 34$ \\
Male : female & $62: 15$ & $35: 5$ \\
Etiology & & \\
$\quad$ HBV & 51 & 24 \\
HCV & 11 & 10 \\
NASH & 5 & 3 \\
Cryptogenic & 4 & 3 \\
$\quad$ Others & 6 & 0 \\
Bilirubin & $1.34 \pm 1.56$ & $1.76 \pm 3.48$ \\
Protein & $3.20 \pm 0.57$ & $3.11 \pm 0.68$ \\
AFP & $27,980 \pm 151,661$ & $4,234 \pm 10,924$ \\
ALT & $60.6 \pm 32.8$ & $56.5 \pm 33.2$ \\
INR & $1.25 \pm 0.21$ & $1.40 \pm 0.85$ \\
PT & $15.56 \pm 2.60$ & $15.71 \pm 4.99$ \\
\hline
\end{tabular}

preceding pathologies prevails. With the advent of technological development in medical science and cancer management, these advanced countries have started to manage HCC patients with an aim to assess 5-year survival after diagnosis.

In Japan, the 5-year and 10-year overall survival (OS) rates in the cohort of 173,378 HCC patients were $37.9 \%$ and $16.5 \%$, respectively. However, over time, the mean maximum tumor size decreased significantly, whereas 5-year OS rates and median survival time increased significantly. ${ }^{12}$ Similar findings were observed separately in patients who underwent resection, local ablation, TACE, and hepatic arterial infusion chemotherapy (HAIC), as well as in patients with AFP levels $\geq 400 \mathrm{ng} / \mathrm{mL}$. In the United States, 2-year survival after surgical treatment was $44 \%$ in a cohort of 11,187 patients with HCC. ${ }^{13}$ In Korea, a cohort of 63 HCC cases (stage I; 1, stage II; 17 , stage III; 38, and stage IV; 7 cases) was treated and the actual 5 -year survival rate was $57.0 \%$ and the median survival time was 60 months. ${ }^{14}$ The prognosis of advanced HCC is extremely bad and it has been reported that these patients may survive some months even getting treatment with sorafenib. ${ }^{15}$

The study presented here has shown that 33 out of 77 patients with advanced HCC died within 3 months and majority of them died within 1 month after therapy commencement. We found that patients with HBV-related HCC seem to have a fatal course, but there have been several related factors for these observations. Although elusive, it might be an important factor as out of 77 patients in this cohort, 44 patients with advanced HCC are surviving 3 months after therapy commencement.

This is a single-tailored study and there is no comparison with any types of control. We are not sure how the patients would behave if they receive only one mode of therapy in spite of both. These points remain to be resolved in future by conducting clinical trials with two or three drug control.

The present study indicates that therapy of advanced HCC (presented in this study) would be a controversial issue, if about $43 \%$ patients die within 3 months and about 21\% die within 1 month after therapy commencement. Special attention should be taken to treat these patients when the $S O L$ is diffuse in nature. This also indicates the importance of HCC surveillance in Bangladesh. It seems that if patients attend the physicians at an early state, almost all sorts of therapy can prolong their overall survival. 


\section{References}

1. Yang JD, Hainaut $P$, Gores GJ, et al. A global view of hepatocellular carcinoma: trends, risk, prevention and management. Nat Rev Gastroenterol Hepatol 2019;16(10):589-604. DOI: 10.1038/s41575019-0186-y.

2. Rawla P, Sunkara T, Muralidharan $\mathrm{P}$, et al. Update in global trends and aetiology of hepatocellular carcinoma. Contemp Oncol (Pozn) 2018;22(3):141-150. DOI: 10.5114/wo.2018.78941.

3. Noor-E-Alam SM. Management of hepatocellular carcinoma: Bangladesh perspective. Euroasian J Hepatogastroenterol 2018;8(1):52-53. DOI: 10.5005/jp-journals-10018-1258.

4. Cahill BA, Braccia D. Current treatment for hepatocellular carcinoma. Clin J Oncol Nurs 2004;8(4):393-399. DOI: 10.1188/04.CJON.393-399.

5. Wege H, Li J, Ittrich $\mathrm{H}$. Treatment lines in hepatocellular carcinoma. Visc Med 2019;35(4):266-272. DOI: 10.1159/000501749.

6. Kew MC. Hepatocellular carcinoma in developing countries: prevention, diagnosis and treatment. World J Hepatol 2012;4(3): 99-104. DOI: 10.4254/wjh.v4.i3.99.

7. Mahtab MA, Chaudhury M, Uddin MH, et al. Cost assessment of hepatitis $B$ virus-related hepatitis in Bangladesh. Euroasian J Hepatogastroenterol 2016;6(2):163-166. DOI: 10.5005/ jp-journals-10018-1190.

8. Chen QF, Wu PH, Huang T, et al. Efficacy of treatment regimens for advanced hepatocellular carcinoma: a network meta-analysis of randomized controlled trials. Medicine (Baltimore) 2019;98(40):e17460. DOI: 10.1097/MD.0000000000017460.
9. Paul SB, Dhamija E, Gamanagatti SR, et al. Evaluation of tumor response to intra-arterial chemoembolization of hepatocellular carcinoma: comparison of contrast-enhanced ultrasound with multiphase computed tomography. Diagn Interv Imaging 2017;98(3):253-260. DOI: 10.1016/j.diii.2016.09.002.

10. Agarwal A, Yadav AK, Kumar A, et al. Transarterial chemoembolization in unresectable hepatocellular carcinoma-assessing the factors affecting the survival: an audit from a tertiary care center in northern India. Indian J Gastroenterol 2015;34(2):117-126. DOI: 10.1007/s12664-015-0544-9.

11. Goel M, Gaikwad V, Dharia T, et al. Preresection transarterial chemoembolization for hepatocellular carcinoma: an experience with 23 patients. Indian J Gastroenterol 2014;33(5):432. DOI: 10.1007/ s12664-014-0490-y.

12. Kudo M, Izumi N, Sakamoto M, et al. Survival analysis over 28 years of 173,378 patients with hepatocellular carcinoma in Japan. Liver Cancer 2016;5(3):190-197. DOI: 10.1159/000367775.

13. Golabi P, Fazel S, Otgonsuren M, et al. Mortality assessment of patients with hepatocellular carcinoma according to underlying disease and treatment modalities. Medicine 2017;96(9):e5904. DOI: 10.1097/ MD.0000000000005904.

14. Li L, Zhao W, Wang M, et al. Transarterial chemoembolization plus sorafenib for the management of unresectable hepatocellular carcinoma: a systematic review and meta-analysis. BMC Gastroenterol 2018;18(1):138. DOI: 10.1186/s12876-018-0849-0.

15. Colagrande S, Inghilesi AL, Aburas S, et al. Challenges of advanced hepatocellular carcinoma. World J Gastroenterol 2016;22(34): 7645-7659. DOI: 10.3748/wjg.v22.i34.7645. 